\title{
Article \\ Emergence of Anyons on the Two-Sphere in Molecular Impurities
}

\author{
Morris Brooks ${ }^{1, *}$, Mikhail Lemeshko ${ }^{1}$, Douglas Lundholm ${ }^{2}$ and Enderalp Yakaboylu ${ }^{1,3, *}$ \\ 1 IST Austria (Institute of Science and Technology Austria), Am Campus 1, 3400 Klosterneuburg, Austria; \\ mikhail.lemeshko@ist.ac.at \\ 2 Department of Mathematics, Uppsala University, P.O. Box 480, SE-751 06 Uppsala, Sweden; \\ douglas.lundholm@math.uu.se \\ 3 Max Planck Institute of Quantum Optics, 85748 Garching, Germany \\ * Correspondence: morris.brooks@ist.ac.at (M.B.); enderalp.yakaboylu@ist.ac.at (E.Y.)
}

check for updates

Citation: Brooks, M.; Lemeshko, M.; Lundholm, D.; Yakaboylu, E. Emergence of Anyons on the Two-Sphere in Molecular Impurities. Atoms 2021, 9, 106. https://doi.org/ 10.3390 /atoms 9040106

Academic Editors: Simeon Mistakidis and Artem Volosniev

Received: 23 August 2021

Accepted: 19 November 2021

Published: 2 December 2021

Publisher's Note: MDPI stays neutral with regard to jurisdictional claims in published maps and institutional affiliations.

Copyright: (c) 2021 by the authors. Licensee MDPI, Basel, Switzerland. This article is an open access article distributed under the terms and conditions of the Creative Commons Attribution (CC BY) license (https:// creativecommons.org/licenses/by/ $4.0 /)$.
Abstract: Recently it was shown that anyons on the two-sphere naturally arise from a system of molecular impurities exchanging angular momentum with a many-particle bath (Phys. Rev. Lett. 126,015301 (2021)). Here we further advance this approach and rigorously demonstrate that in the experimentally realized regime the lowest spectrum of two linear molecules immersed in superfluid helium corresponds to the spectrum of two anyons on the sphere. We develop the formalism within the framework of the recently experimentally observed angulon quasiparticle.

Keywords: anyons; quasiparticles; Quantum Hall Effect; topological states of matter

\section{Introduction}

The discovery of the fractional Quantum Hall Effect and the advent and application of topological quantum field theories have revolutionized our understanding of the quantum properties of matter [1-8]. Among the prospective applications, the notion of topological quantum computation has recently emerged as one of the most exciting approaches for constructing a fault-tolerant quantum computer by seeking to exploit the emergent properties of many-particle systems to encode and manipulate quantum information in a manner which is resistant to error [9-12]. One simple such proposal for topological quantum computing and information storage relies on the existence of topological states of matter whose quasiparticle excitations are anyons.

An anyon is a type of quasiparticle that can arise in systems confined to two dimensions and whose exchange properties interpolate between bosons and fermions [13-15]. Because of the topological peculiarities of two spatial dimensions, the world lines of anyons can braid nontrivially around each other $[13,16,17]$, and therefore, unlike fermions or bosons, exchanging two anyons twice is not topologically equivalent to leaving them alone. This opens up a whole new domain of quantum statistics known as intermediate or fractional statistics. Even though the realization of anyons in experimentally feasible systems has been subject of recent research [18-25], all these works concern particles moving on the Euclidean plane $\mathbb{R}^{2}$, or a subset thereof. However, since the statistical behaviour of anyons depends on the topology, and even more importantly on the geometry and symmetry, of the underlying space, investigations on curved spaces can demonstrate novel properties of quantum statistics [2,26-32] (see also graph geometries [33,34]). Indeed it has been recently demonstrated that the emerging fractional statistics for particles restricted to move on the sphere, instead of on the plane, arises naturally in the context of quantum impurity problems, particularly, in the context of molecular impurities [35]. There, it has been shown that the emerging statistical interaction manifests itself in the alignment of molecules, which could also be of use as a powerful technique to measure the statistics parameter. This paves the way towards experimental realization as well as detection of anyons on the sphere using molecular impurities. 
In the present manuscript, we explicitly show how the angulon Hamiltonian [36-38] gives rise to a system of two interacting anyons on the two-sphere $\mathbb{S}^{2}$. The angulon represents a quantum impurity exchanging orbital angular momentum with a many-particle bath, and serves as a reliable model for the rotation of molecules in superfluids [39-42]. In particular, we demonstrate that, under appropriate time-reversal symmetry breaking conditions, restricting the angulon Hamiltonian to states in the first Born-Oppenheimer approximation gives rise to the anyon Hamiltonian. Time-reversal symmetry is broken by using an additional external magnetic field and applying rotation, while the Born-Oppenheimer approximation is satisfied by considering heavy molecules. We further discuss and supply some technical details of the argument that had been left out in [35]. Note that the phenomenon of quantum statistics transmutation typically involves emergent scalar interaction potentials and non-statistical gauge fields as well, and it is necessary to have sufficient control of these effects in order to provide robust signatures of anyons.

\section{Anyon Hamiltonian}

Anyons are identical particles described by wave functions $\Psi$ which acquire a phase factor $e^{i \alpha \pi}$, respectively $e^{-i \alpha \pi}$, under permutation of two sets of coordinates. In contrast to fermions and bosons, we do not assume that the statistics parameter $\alpha$ is an integer. Namely, it could be any real number, say between 0 and 1, or between -1 and 0 , i.e., a fraction of an integer (thereby 'fractional statistics'). Consequently, we have to distinguish between the continuous exchange processes where two particles make an elementary anti-clockwise braid around each other, in which case the wave function gains a factor $e^{i \alpha \pi}$, and processes where they braid clockwise around each other, in which case the wave function has to acquire the inverse factor $e^{-i \alpha \pi}$. Here we however see a difference on the sphere compared to the plane, since e.g., the 2-particle braid group reduces from $\mathbb{Z}$ on the plane to $\mathbb{Z}_{2}$ on the sphere, due to a double exchange being topologically trivial. This also means that we cannot determine topologically which way the particles braided, and thus reduces the whole problem to the ordinary case of bosons or fermions, $\alpha \in\{0,1\}$. In fact, this conclusion is a manifestation of the symmetry of the full sphere, and indeed the existence of anyons necessarily requires the breaking of time-reversal or orientation symmetry (corresponding to the choice of sign of $\alpha$ and the handedness of braids in our braid group representation). A similar analysis for the $N$-particle case leads to the condition $(N-1) \alpha \in \mathbb{Z}$, analogous to the well-known Dirac quantization condition $[26,28,30]$. We can overcome this issue, by instead considering the punctured sphere $\mathbb{S}^{2} \backslash\{\mathcal{N}\}$, where $\mathcal{N}$ denotes the north pole (and $\mathcal{S}$ will denote the south pole), i.e., we consider anyons which are no longer invariant under the action of $O(3)$ but only with respect to rotations in the polar angle. Clearly, $\mathbb{S}^{2} \backslash\{\mathcal{N}\}$ is topologically equivalent to the plane. Nevertheless, the analysis of anyons living on the sphere (or a subset thereof) requires novel ideas and techniques. The first reason for this is that $\mathbb{S}^{2} \backslash\{\mathcal{N}\}$ carries a non-flat geometry, i.e., the free dynamics of two anyons is given by the Hamiltonian

$$
H_{\text {Anyon }}^{\text {sing }}:=-\sum_{j=1}^{2} \nabla_{j}^{2}:=-\sum_{j=1}^{2} g_{a b} \nabla_{j}^{a} \nabla_{j}^{b},
$$

where $g_{a b}$ is the metric tensor of the sphere, and we put suitable conditions at $\mathcal{N}$ and on the coincidence set for the particles (for simplicity, we may consider functions $\Psi$ vanishing on the diagonal of the configuration space $\left(\mathbb{S}^{2} \backslash\{\mathcal{N}\}\right)^{2}$; cf. [43-45].) The second difference to the plane is that the natural orientation-preserving symmetry group of the full sphere is given by the three dimensional rotations $S O(3)$, while the symmetry group of the plane consists of a rotation around a single axis and translations in the plane. As one might expect, and we will see explicitly below, the symmetry group plays a crucial role in deriving the emergence of anyons from suitable impurity problems. 
It will be convenient to represent the anyonic wave function as $\Psi=e^{i \alpha \phi} \psi$, where $\psi$ is a bosonic wave function and $\phi$ is a fixed smooth multivalued function with the property $\phi\left(q_{2}, q_{1}\right)=\phi\left(q_{1}, q_{2}\right) \pm \pi$ under simple continuous exchange of the two coordinates $q_{j} \in \mathbb{S}^{2}$, in order that $\Psi$ acquires a correct phase factor $e^{ \pm i \alpha \pi}$. A concrete example of such a function $\phi$ is given in complex stereographic coordinates $z_{1}, z_{2} \in \mathbb{C}$ by $\frac{1}{i} \log \left(\frac{z_{1}-z_{2}}{\left|z_{1}-z_{2}\right|}\right)$. Applying the unitary transformation $e^{i \alpha \phi}$ to the free anyon dynamics yields

$$
H_{\text {Anyon }}:=e^{-i \alpha \phi} H_{\text {Anyon }}^{\text {sing }} e^{i \alpha \phi}=-\sum_{j=1}^{2}\left(\nabla_{j}+i \alpha \nabla_{j} \phi\right)^{2}=-\sum_{j=1}^{2}\left(\nabla_{j}+i A_{j}\right)^{2},
$$

with the anyon statistics gauge field $A_{j}$ given by $A_{j}:=\alpha \nabla_{j} \phi$. Note that $H_{\text {Anyon, which is }}$ unitarily equivalent to the free anyon dynamics (although by a singular gauge transformation, thereby changing the reference geometry) has the advantage of acting on bosonic (single-valued) wave functions $\psi$.

\section{Emerging Gauge Field from the Angulon Hamiltonian}

The angulon Hamiltonian for two rotors/impurities is defined by

$$
H_{\text {angulon }}:=-\sum_{j=1}^{2} \nabla_{j}^{2}+\sum_{l, m} \omega_{l} b_{l, m}^{\dagger} b_{l, m}+b_{Z\left(q_{1}, q_{2}\right)}^{+}+b_{Z\left(q_{1}, q_{2}\right)},
$$

where $-\nabla_{j}^{2}=\mathbf{L}_{j}^{2}=L_{j x}^{2}+L_{j y}^{2}+L_{j z}^{2}$ is the rotor Hamiltonian, $q_{j} \in \mathbb{S}^{2}$ is the position of the $j$-th impurity on the sphere, $b_{l, m}$ are collective rotation modes of the bath, and $b_{Z\left(q_{1}, q_{2}\right)}^{(\dagger)}=\sum_{l, m} Z_{l, m}\left(q_{1}, q_{2}\right)^{(*)} b_{l, m}^{(\dagger)}$ defines the coupling between these systems at the Fröhlich level [36-38]. Note that this Hamiltonian is typically fully invariant under the action of $O(3)$, so that we cannot expect any non-trivial anyons to emerge.

Instead, in the following, we aim to derive the statistics gauge field $A_{j}$ as emergent from the following modified angulon Hamiltonian:

$$
H_{\text {angulon }, \Omega}^{\prime}:=H_{\text {angulon }}+\Omega^{2} V\left(q_{1}, q_{2}\right)+\Omega \sum_{l, m_{1}, m_{2}}\left(\Lambda_{\bar{q}}\right)_{l, m_{1}, m_{2}} b_{l, m_{1}}^{\dagger} b_{l, m_{2}},
$$

where $\bar{q}:=\left(q_{1}+q_{2}\right) /\left|q_{1}+q_{2}\right|$ is the normalized center of mass of the two impurities,

$$
\left(\Lambda_{\bar{q}}\right)_{l, m_{1}, m_{2}}:=\sum_{m} m \overline{D_{m, m_{2}}^{l}(\alpha, \beta, \gamma)} D_{m, m_{1}}^{l}(\alpha, \beta, \gamma)
$$

is the momentum operator aligned in the direction of $-\bar{q}$ which we define with the help of the Wigner matrix $D_{m, m_{2}}^{l}(\alpha, \beta, \gamma)$ where $\alpha, \beta, \gamma$ are the Euler angles of a rotation $R_{\alpha, \beta, \gamma}$ with the property $R_{\alpha, \beta, \gamma}(\mathcal{S})=\bar{q}, V$ is an additional quadratic potential, and the parameter $\Omega$, which will describe the strength of a simultaneous magnetic field and a rotation, is assumed to be large. With the convention above, the momentum operator $\Lambda_{z}$ aligned with the $z$-axis reads $\Lambda_{z}=\Lambda_{\mathcal{S}}$. Note that having the momentum operator $\Lambda_{\bar{q}}$ aligned in the direction $\bar{q}$ will simplify our computation significantly. In the next section we will discuss a model where we take the operator $\Lambda_{z}=\Lambda_{\mathcal{S}}$ aligned with the $z$-axis as usual, and argue that as $\Omega \rightarrow \infty$ they describe the same limit within a certain setup. We will also discuss how one can realize the modified operator $H_{\text {angulon, },}^{\prime}$, by coupling $H_{\text {angulon }}$ to an additional constant magnetic field. In this concrete realization of Equation (3) the scaling on $V$ comes naturally.

We refer to Hamiltonian (3) as modified, since it has a dispersion relation $\sum_{l, m} \omega_{l} b_{l, m}^{\dagger} b_{l, m}+\Omega \sum_{l, m_{1}, m_{2}}\left(\Lambda_{\bar{q}}\right)_{l, m_{1}, m_{2}} b_{l, m_{1}}^{\dagger} b_{l, m_{2}}$ which is not invariant under a change of orientation. Furthermore, the introduction of a suitably chosen potential $V$ punctures the sphere and therefore breaks the $S O(3)$ invariance as well. Let us denote with $\theta_{j}$ the 
azimuthal angle of the impurity position $q_{j}$ and with $\varphi_{j}$ its polar angle w.r.t. the laboratory reference frame. The $q_{1}, q_{2}$-dependent coefficients of $Z\left(q_{1}, q_{2}\right)$ are then given by

$$
Z_{l, m}:=\sum_{j} c_{l} Y_{l, m}\left(\theta_{j}, \varphi_{j}\right)
$$

where $Y_{l, m}$ are the spherical harmonics and $c_{l}$ are real coefficients. We will occasionally suppress the $q_{1}, q_{2}$-dependency of $Z\left(q_{1}, q_{2}\right)$, and simply write $Z$. Note that we may instead of Equation (3) consider a symmetry-breaking interaction such as $\tilde{Z}:=$ $\left(1+\Omega \Lambda_{\bar{q}} \omega^{-1}\right)^{-1} Z\left(q_{1}, q_{2}\right)$ leading to the emergence of anyons with the same statistical gauge field. As stated, however, we here aim for a simplest possible realization of anyons, as a first step.

The full Hamiltonian (3) acts on an appropriate dense domain in the tensor product Hilbert space of the impurities $L_{\text {sym /asym }}^{2}\left(\mathbb{S}^{2} \times \mathbb{S}^{2}\right)$, where $L_{\text {sym }}^{2}\left(\mathbb{S}^{2} \times \mathbb{S}^{2}\right)$ is the bosonic Hilbert space and $L_{\text {asym }}^{2}\left(\mathbb{S}^{2} \times \mathbb{S}^{2}\right)$ the fermionic one, with the Fock space $\mathcal{F}\left(L^{2}\left(\mathbb{S}^{2}\right)\right)$ of the bath. Following the analysis for impurity problems in the planar case [25], the statistics gauge field emerges from $H_{\text {angulon, },}^{\prime}$, by restricting it to the ground state of its pure manybody part $\sum_{l, m} \omega_{l} b_{l, m}^{\dagger} b_{l, m}+\Omega \sum_{l, m_{1}, m_{2}}\left(\Lambda_{\bar{q}}\right)_{l, m_{1}, m_{2}} b_{l, m_{1}}^{\dagger} b_{l, m_{2}}+b_{Z\left(q_{1}, q_{2}\right)}^{+}+b_{Z\left(q_{1}, q_{2}\right)}$ which acts only on the Fock space $\mathcal{F}\left(L^{2}\left(\mathbb{S}^{2}\right)\right)$ of the bath. Namely, with the help of a coherent state transformation, we can write the ground state as (we use the notation $b_{x}=\sum_{l, m} x_{l m} b_{l m}$ and : for action or composition)

$$
\Phi\left(q_{1}, q_{2}\right):=\exp \left[b_{\left(\omega+\Omega \Lambda_{\bar{q}}\right)^{-1} Z\left(q_{1}, q_{2}\right)}-b_{\left(\omega+\Omega \Lambda_{\bar{q}}\right)^{-1} Z\left(q_{1}, q_{2}\right)}^{+}\right] \cdot|0\rangle .
$$

Explicitly, by completing the square,

$$
b^{\dagger} \cdot\left(\omega+\Omega \Lambda_{\bar{q}}\right) \cdot b+b_{Z}^{\dagger}+b_{Z}=(b+\xi)^{\dagger} \cdot\left(\omega+\Omega \Lambda_{\bar{q}}\right) \cdot(b+\xi)-c,
$$

with $\xi:=\left(\omega+\Omega \Lambda_{\bar{q}}\right)^{-1} Z$ and $c:=\xi^{\dagger}\left(\omega+\Omega \Lambda_{\bar{q}}\right) \xi$. We see that the non-symmetric dispersion relation $\omega+\Omega \Lambda_{\bar{q}}$ leads to a breaking of symmetry in the vacuum section $\Phi\left(q_{1}, q_{2}\right)$, since the coefficients $\left(\omega+\Omega \Lambda_{\bar{q}}\right)^{-1} \cdot Z\left(q_{1}, q_{2}\right)$ are no longer invariant under the action of $O(3)$.

In the following, we consider a gapped dispersion $\omega_{l} \rightarrow \infty$ and heavy impurities such that the ground state decouples from the rest of the Hamiltonian. In this regime, the low energy spectrum of Hamiltonian (3) can be described by the first Born-Oppenheimer approximation

$$
\left\langle\psi\left|H_{\text {Emerg }}\right| \psi\right\rangle:=\left\langle\psi \Phi\left|H_{\text {angulon }, \Omega}^{\prime}\right| \psi \Phi\right\rangle,
$$

where $\psi\left(q_{1}, q_{2}\right)$ is an impurity wave function (bosonic or fermionic). By applying the coherent state transformation $S_{0}:=\exp \left[b_{\left(\omega+\Omega \Lambda_{\bar{q}}\right)^{-1} Z\left(q_{1}, q_{2}\right)}-b_{\left(\omega+\Omega \Lambda_{\bar{q}}\right)^{-1} Z\left(q_{1}, q_{2}\right)}^{+}\right]$as above, we see that, formally,

$$
H_{\text {Emerg }}=\left\langle 0\left|-\sum_{j}\left(\nabla_{j}+S_{0}^{-1}\left(\nabla_{j} S_{0}\right)\right)^{2}\right| 0\right\rangle+\Omega^{2} V-Z^{\dagger}\left(\omega+\Omega \Lambda_{\bar{q}}\right)^{-1} Z .
$$

The issue with this representation is that we do not have a nice expression for the quantity $S_{0}^{-1} \nabla_{j} S_{0}$. This is due to the fact that the following family of operators is non-commuting:

$$
\left\{b_{\left(\omega+\Omega \Lambda_{\bar{q}}\right)^{-1} Z\left(q_{1}, q_{2}\right)}-b_{\left(\omega+\Omega \Lambda_{\bar{q}}\right)^{-1} Z\left(q_{1}, q_{2}\right)}^{\dagger}: q_{1}, q_{2} \in \mathbb{S}^{2}\right\},
$$

and therefore we cannot apply the usual chain rule $\nabla_{j} \exp [F]=\nabla_{j} F \exp [F]$. In order to arrive at an explicit expression, we will apply two unitary transformations, which should map the non-commuting family to a commuting one. Note that this transformation has to be 
$q_{1}, q_{2}$-dependent, since a single fixed unitary transformation always maps non-commuting families to non-commuting ones.

We first need to transform the whole system to a fixed reference point, such that $\bar{q} \mapsto \bar{q}^{\prime}=\mathcal{S}$, i.e., such that the middlepoint $\bar{q}$ between $q_{1}$ and $q_{2}$ stays fixed at the south pole $\mathcal{S}$. For an arbitrary position $q \neq \mathcal{N}$ which is not the north pole, let $T \in S O(3)$ be a rotation which maps $q$ into the south pole, i.e., $T(q)=\mathcal{S}$. Clearly there are many rotations which satisfy $T(q)=\mathcal{S}$. Therefore, we demand further that $T$ leaves the axis $\mathcal{S} \times q$ invariant for $q \neq \mathcal{S}$ and define $T$ to be the identity if $q=\mathcal{S}$. The conditions $T(q)=\mathcal{S}$ and $T(\mathcal{S} \times q)=\mathcal{S} \times q$ uniquely determine the map $T$. Since $T$ is $q$-dependent, we will write $T_{q}=T$. In the following, we will always use the center of mass $\bar{q}$ as the argument, i.e., we consider $T_{\bar{q}}$. In order to promote $T_{\bar{q}}$ to a transformation on the whole Hilbert space, note that we can write it as

$$
T_{\bar{q}}=\exp \left[\left(\begin{array}{rrr}
0 & -z_{\bar{q}} & y_{\bar{q}} \\
z_{\bar{q}} & 0 & -x_{\bar{q}} \\
-y_{\bar{q}} & x_{\bar{q}} & 0
\end{array}\right)\right],
$$

with coefficients $\left(x_{\bar{q}}, y_{\bar{q}}, z_{\bar{q}}\right):=d(\bar{q}, \mathcal{S})(\mathcal{S} \times \bar{q}) /|\mathcal{S} \times \bar{q}|$, where $d(\bar{q}, \mathcal{S})$ is the geodesic distance of $\bar{q}$ to the south pole $\mathcal{S}$. Let us furthermore denote transformed points as $q^{\prime}:=T_{\bar{q}} \cdot q$. With this at hand, we can define the transformation of a Fock space valued state $\Psi\left(q_{1}, q_{2}\right)$ as

$$
\hat{T}(\Psi)\left(q_{1}, q_{2}\right):=\exp \left[i b^{\dagger} \cdot\left(x_{\bar{q}} \Lambda_{x}+y_{\bar{q}} \Lambda_{y}+z_{\bar{q}} \Lambda_{z}\right) \cdot b\right] \cdot \Psi\left(q_{1}, q_{2}\right) .
$$

Recall that the transformation $T_{\bar{q}}$ only makes sense as long as $\bar{q} \neq \mathcal{N}$. Therefore, we only consider this transformation $\hat{T}$ for confined states $\Psi$, for example only for states which have a support contained in an open set $O \subset \bar{O} \subset\left\{q \in \mathbb{S}^{2}: q_{3}<0\right\}$. Note that this is not necessarily a real restriction, since the modified operator $H_{\text {angulon }, \Omega}^{\prime}$ contains a confining potential $V$ anyway, which we will assume to have its minimum close to $\mathcal{S}$.

We can write the transformed Hamiltonian $\hat{T}^{-1} H_{\text {angulon, } \Omega}^{\prime} \hat{T}$ as

$$
-\sum_{j}\left(\nabla_{j}+\hat{T}^{-1}\left(\nabla_{j} \hat{T}\right)\right)^{2}+\Omega^{2} V\left(q_{1}, q_{2}\right)+b^{\dagger} \cdot\left(\omega+\Omega \Lambda_{z}\right) \cdot b+b_{Z\left(q_{1}^{\prime}, q_{2}^{\prime}\right)}^{+}+b_{Z\left(q_{1}^{\prime}, q_{2}^{\prime}\right)} .
$$

Note that after the transformation, the angular momentum operator $\Lambda_{z}$ is aligned with respect to the $z$-axis instead of the direction $\bar{q}$. Let us denote with $\phi=\phi\left(q_{1}, q_{2}\right)$ the polar angle of $q_{1}^{\prime}$, which is the position of the first impurity after the rotation $T$. Furthermore, let $R=R_{\phi}$ be a rotation around the $z$-axis by an amount of $\phi$. Then, the polar angle of the transformed point $q_{1}^{\prime \prime}:=R^{-1}\left(q_{1}^{\prime}\right)$ is zero, while the polar angle of $q_{2}^{\prime \prime}:=R^{-1}\left(q_{2}^{\prime}\right)$ equals $\pi$. Both points $q_{1}^{\prime \prime}, q_{2}^{\prime \prime}$ have the same azimuthal angle $\theta$. We promote $R=R_{\phi}$ to an operation on the whole Hilbert space by

$$
\hat{R}(\Psi)\left(q_{1}, q_{2}\right):=\exp \left[i \phi b^{\dagger} \cdot \Lambda_{z} \cdot b\right] \cdot \Psi\left(q_{1}, q_{2}\right) .
$$

The transformed operator $\hat{R}^{-1} \hat{T}^{-1} H_{\text {angulon, } \Omega}^{\prime} \hat{T} \hat{R}$ then reads

$$
\begin{aligned}
& -\sum_{j}\left(\nabla_{j}+\hat{R}^{-1} \hat{T}^{-1}\left(\nabla_{j} \hat{T}\right) \hat{R}+i\left(\nabla_{j} \phi\right) \sum_{l, m} m b_{l, m}^{+} b_{l, m}\right)^{2}+\Omega^{2} V\left(q_{1}, q_{2}\right)+b^{\dagger} \cdot\left(\omega+\Omega \Lambda_{z}\right) \cdot b \\
& \quad+b_{Z\left(q_{1}^{\prime \prime}, q_{2}^{\prime \prime}\right)}^{+}+b_{Z\left(q_{1}^{\prime \prime}, q_{2}^{\prime \prime}\right)},
\end{aligned}
$$


In the final step, we diagonalize the pure many body part $b^{\dagger} \cdot\left(\omega+\Omega \Lambda_{z}\right) \cdot b+b_{Z\left(q_{1}^{\prime \prime}, q_{2}^{\prime \prime}\right)}^{+}+$ $b_{Z\left(q_{1}^{\prime \prime}, q_{2}^{\prime \prime}\right)}$, by applying the coherent state transformation

$$
S:=\exp \left[b_{\left(\omega+\Omega \Lambda_{z}\right)^{-1} Z\left(q_{1}^{\prime \prime}, q_{2}^{\prime \prime}\right)}-b_{\left(\omega+\Omega \Lambda_{z}\right)^{-1} Z\left(q_{1}^{\prime \prime}, q_{2}^{\prime \prime}\right)}^{\dagger}\right] .
$$

Since the coefficients at the transformed points $Z_{l, m}\left(q_{1}^{\prime \prime}, q_{2}^{\prime \prime}\right)=\left(1+(-1)^{m}\right) c_{l} Y_{l, m}(\theta, 0)$ are all real valued and the expressions only dependent on $\theta$, we know that the collection

$$
\left\{b_{\left(\omega+\Omega \Lambda_{z}\right)^{-1} Z\left(q_{1}^{\prime \prime}, q_{2}^{\prime \prime}\right)}-b_{\left(\omega+\Omega \Lambda_{z}\right)^{-1} Z\left(q_{1}^{\prime \prime}, q_{2}^{\prime \prime}\right)}^{\dagger}: q_{1}, q_{2} \in O\right\}
$$

is a family of commuting operators. Consequently, we can finally apply the chain rule and compute $S^{-1}\left(\nabla_{j} S\right)$ quite explicitly as

$$
S^{-1}\left(\nabla_{j} S\right)=b_{\left(\omega+\Omega \Lambda_{z}\right)^{-1} \nabla_{j} Z\left(q_{1}^{\prime \prime}, q_{2}^{\prime \prime}\right)}-b_{\left(\omega+\Omega \Lambda_{z}\right)^{-1} \nabla_{j} Z\left(q_{1}^{\prime \prime}, q_{2}^{\prime \prime}\right)}^{\dagger} .
$$

We can express the transformed Hamiltonian $S^{-1} \hat{R}^{-1} \hat{T}^{-1} H_{\text {angulon, } \Omega}^{\prime} \hat{T} \hat{R} S$ as

$$
\begin{aligned}
-\sum_{j}( & \left.\nabla_{j}+i \alpha(\theta)\left(\nabla_{j} \phi\right)+Y_{j}+S^{-1}\left(\nabla_{j} S\right)-i\left(\nabla_{j} \phi\right)\left(b_{W}+b_{W}^{+}\right)+i\left(\nabla_{j} \phi\right) \sum_{l, m} m b_{l, m}^{+} b_{l, m}\right)^{2} \\
& +\Omega^{2} V\left(q_{1}, q_{2}\right)-E_{0}+b^{\dagger} \cdot\left(\omega+\Omega \Lambda_{z}\right) \cdot b
\end{aligned}
$$

with the abbreviations

$$
\begin{gathered}
W:=\Lambda_{z}\left(\omega+\Omega \Lambda_{z}\right)^{-1} Z\left(q_{1}^{\prime \prime}, q_{2}^{\prime \prime}\right), \\
Y_{j}:=S^{-1} \hat{R}^{-1} \hat{T}^{-1}\left(\nabla_{j} \hat{T}\right) \hat{R} S, \\
E_{0}:=Z\left(q_{1}^{\prime \prime}, q_{2}^{\prime \prime}\right)^{T}\left(\omega+\Omega \Lambda_{z}\right)^{-1} Z\left(q_{1}^{\prime \prime}, q_{2}^{\prime \prime}\right),
\end{gathered}
$$

and

$$
\alpha(\theta):=Z\left(q_{1}^{\prime \prime}, q_{2}^{\prime \prime}\right)^{T}\left(\omega+\Omega \Lambda_{z}\right)^{-1} \Lambda_{z}\left(\omega+\Omega \Lambda_{z}\right)^{-1} Z\left(q_{1}^{\prime \prime}, q_{2}^{\prime \prime}\right) .
$$

Observe that the vacuum expectation of $i \nabla_{j} \phi\left(b_{W}+b_{W}^{\dagger}\right)$ and $i \nabla_{j} \phi \sum_{l, m} m b_{l, m}^{+} b_{l, m}$ is zero. Therefore these terms will only contribute to the emergent scalar potential but not to the emergent gauge field. Let us recall the definition of the vacuum section $\Phi$ in Equation (4). With the help of the unitary maps $T, R$ and $S$ we can write $\Phi=\hat{T} \hat{R} S|0\rangle$ and consequently

$$
\begin{aligned}
H_{\text {Emerg }} & :=\left\langle\Phi\left|H_{\text {angulon, } \Omega}^{\prime}\right| \Phi\right\rangle=\left\langle 0\left|S^{-1} \hat{R}^{-1} \hat{T}^{-1} H_{\text {angulon }, \Omega}^{\prime} \hat{T} \hat{R} S\right| 0\right\rangle \\
& =-\langle 0| \sum_{j}\left(\nabla_{j}+i \alpha(\theta)\left(\nabla_{j} \phi\right)+Y_{j}+S^{-1}\left(\nabla_{j} S\right)-i\left(\nabla_{j} \phi\right)\left(b_{W}+b_{W}^{\dagger}\right)\right. \\
& \left.+i\left(\nabla_{j} \phi\right) \sum_{l, m} m b_{l, m}^{\dagger} b_{l, m}\right)^{2}|0\rangle+\left\langle 0\left|\Omega^{2} V-E_{0}+b^{\dagger} \cdot\left(\omega+\Omega \Lambda_{z}\right) \cdot b\right| 0\right\rangle .
\end{aligned}
$$

The second expectation is simply $\left\langle 0\left|\Omega^{2} V-E_{0}+b^{\dagger} \cdot\left(\omega+\Omega \Lambda_{z}\right) \cdot b\right| 0\right\rangle=\Omega^{2} V-E_{0}$. In order to compute the first one, let us define the magnetic potential $A_{j}:=\alpha(\theta) \nabla_{j} \phi$ and the gauge covariant derivative $\nabla_{j}^{A}:=\nabla_{j}+i A_{j}$. Let us further abbreviate

$$
U_{j}:=\left(\omega+\Omega \Lambda_{z}\right)^{-1}\left[\nabla_{j} Z\left(q_{1}^{\prime \prime}, q_{2}^{\prime \prime}\right)-i \nabla_{j} \phi \Lambda_{z} Z\left(q_{1}^{\prime \prime}, q_{2}^{\prime \prime}\right)\right] \text {. }
$$


Then we can rewrite the first vacuum expectation as

$$
\begin{aligned}
& \left\langle 0\left|-\sum_{j}\left(\nabla_{j}^{A}+Y_{j}+\left(b_{U_{j}}+b_{U_{j}}^{+}\right)+i\left(\nabla_{j} \phi\right) \sum_{l, m} m b_{l, m}^{+} b_{l, m}\right)^{2}\right| 0\right\rangle \\
& =-\sum_{j}\left[\left(\nabla_{j}^{A}+\left\langle 0\left|Y_{j}\right| 0\right\rangle\right)^{2}-\left\langle 0\left|Y_{j}\right| 0\right\rangle^{2}+\left\langle 0\left|\left(Y_{j}+b_{U_{j}}+b_{U_{j}}^{+}+i\left(\nabla_{j} \phi\right) \sum_{l, m} m b_{l, m}^{+} b_{l, m}\right)^{2}\right| 0\right\rangle\right] .
\end{aligned}
$$

Let us define the magnetic background potential $\tilde{A}_{j}:=-i\left\langle 0\left|Y_{j}\right| 0\right\rangle$ and the modified scalar potential

$$
\widetilde{V}:=\Omega^{2} V+\sum_{j}\left\langle 0\left|Y_{j}\right| 0\right\rangle^{2}-\sum_{j}\left\langle 0\left|\left(Y_{j}+b_{U_{j}}+b_{U_{j}}^{+}+i\left(\nabla_{j} \phi\right) \sum_{l, m} m b_{l, m}^{+} b_{l, m}\right)^{2}\right| 0\right\rangle-E_{0},
$$

then we can compactly express the emergent Hamiltonian as

$$
H_{\text {Emerg }}=-\sum_{j}\left(\nabla_{j}^{A}+i \tilde{A}_{j}\right)^{2}+\widetilde{V}
$$

In case of constant $\alpha(\theta)=\alpha$, the operator $-\sum_{j}\left(\nabla_{j}^{A}\right)^{2}$ corresponds to the anyon Hamiltonian (1) with statistics parameter $\alpha$, i.e.,

$$
H_{\text {Emerg }}=H_{\text {Anyon }}+\sum_{j}\left(\tilde{A}_{j}^{2}-i\left(\nabla_{j} \cdot \tilde{A}_{j}\right)-2 i \tilde{A}_{j} \cdot \nabla_{j}^{A}\right)+\widetilde{V} .
$$

In reference [35], approximately constant $\alpha$ (depending on $\Omega$ ) is indeed realized for a suitable, and experimentally feasible, choice of $\omega$ and $c_{l}$. Particularly, $\omega$ is chosen at the roton minimum of the dispersion relation of superfluid helium, which allows us to achieve a gapped dispersion, and the coupling $c_{l}$ is described by the model interaction used in order to describe angulon instabilities and oscillations observed in the experiment. Therefore, the Hamiltonian $H_{\text {angulon, } \Omega}^{\prime}$ gives rise to a system of two anyons, coupled to an additional magnetic potential $\widetilde{A}_{j}$ and an additional scalar potential $\widetilde{V}$.

In the following let us verify that the magnetic potential $\tilde{A}_{j}$ is regular, which on a suitable scale means that curl $\tilde{A}_{j}$ can be treated as a background field and thus does not influence the statistics. First of all, we can write it as

$$
\tilde{A}_{j}=-i\left\langle 0\left|S^{\dagger} \hat{R}^{\dagger} \hat{T}^{-1} \nabla_{j}(\hat{T}) \hat{R} S\right| 0\right\rangle=-i\left\langle\Phi\left|\hat{T}^{-1} \nabla_{j}(\hat{T})\right| \Phi\right\rangle,
$$

where $\Phi$ is as usual the vacuum section. First we want to compute the Lie algebra element $T_{\bar{q}}^{-1}\left(\nabla_{j} T_{\bar{q}}\right)$. In order to verify that $T_{\bar{q}}$ is a matrix-valued $C^{\infty}$ (smooth) function, so especially that its derivative exists and is a continuous function, recall the explicit representation

$$
T_{\bar{q}}=\exp \left[\frac{d(\bar{q}, \mathcal{S})}{|\mathcal{S} \times \bar{q}|}\left(\begin{array}{rrr}
0 & -(\mathcal{S} \times \bar{q})_{3} & (\mathcal{S} \times \bar{q})_{2} \\
(\mathcal{S} \times \bar{q})_{3} & 0 & -(\mathcal{S} \times \bar{q})_{1} \\
-(\mathcal{S} \times \bar{q})_{2} & (\mathcal{S} \times \bar{q})_{1} & 0
\end{array}\right)\right] .
$$

As long as $\mathcal{S} \times \bar{q} \neq 0$, i.e., as long as $\bar{q} \neq \mathcal{S}$ and $\bar{q} \neq \mathcal{N}, T_{\bar{q}}$ is clearly $C^{\infty}$. Since we want to investigate the limit $q_{1}, q_{2} \rightarrow \mathcal{S}$ anyway, we do not have to worry about the case $\bar{q}=\mathcal{N}$. Regarding the south pole itself, observe that the function $d(\bar{q}, \mathcal{S}) /|\mathcal{S} \times \bar{q}|$ is $C^{\infty}$, even for $\bar{q}=\mathcal{S}$. Consequently, we know that $T_{\bar{q}}^{-1}\left(\nabla_{j} T_{\bar{q}}\right)$ exists and it is a smooth function as long 
as $\bar{q} \neq \mathcal{N}$. Note that $T_{\bar{q}}^{-1}\left(\nabla_{j} T_{\bar{q}}\right)$ is an element of the Lie algebra of $S O(3)$, and therefore we can write it as

$$
T_{\bar{q}}^{-1}\left(\nabla_{j} T_{\bar{q}}\right)=\left(\begin{array}{rrr}
0 & -\gamma\left(q_{1}, q_{2}\right) & \beta\left(q_{1}, q_{2}\right) \\
\gamma\left(q_{1}, q_{2}\right) & 0 & -\alpha\left(q_{1}, q_{2}\right) \\
-\beta\left(q_{1}, q_{2}\right) & \alpha\left(q_{1}, q_{2}\right) & 0
\end{array}\right),
$$

with continuous and real functions $\alpha\left(q_{1}, q_{2}\right), \beta\left(q_{1}, q_{2}\right)$ and $\gamma\left(q_{1}, q_{2}\right)$. Consequently, we can write the operator $\hat{T}^{-1}\left(\nabla_{j} \hat{T}\right)$ as

$$
\hat{T}^{-1}\left(\nabla_{j} \hat{T}\right)=b^{\dagger} \cdot\left(\alpha_{j}\left(q_{1}, q_{2}\right) i \Lambda_{x}+\beta_{j}\left(q_{1}, q_{2}\right) i \Lambda_{y}+\gamma_{j}\left(q_{1}, q_{2}\right) i \Lambda_{z}\right) \cdot b .
$$

From the representation above we see that the additional magnetic field $\tilde{A}_{j}$ is regular and therefore does not contribute to the statistics, i.e., $H_{\text {Emerg }}$ describes anyons subject to an additional magnetic gauge field $\tilde{A}_{j}\left(q_{1}, q_{2}\right)$ as well as an additional electric potential field $\widetilde{V}\left(q_{1}, q_{2}\right)$. Let us now describe a set-up, where the additional magnetic background field $\tilde{A}=\left(\tilde{A}_{1}, \tilde{A}_{2}\right)$ can be neglected entirely, i.e., we look for reasonable conditions such that $\tilde{A} \underset{q_{1}, q_{2} \rightarrow \mathcal{S}}{\longrightarrow} 0$. Since $T$ and $\Phi$ are compatible with rotations around the $z$ axis, we know that $\tilde{A}\left(q_{1}=\mathcal{S}, q_{2}=\mathcal{S}\right)=0$. It is therefore enough to verify that the vector field $\tilde{A}$ is continuous. By our representation of $\hat{T}^{-1}\left(\nabla_{j} \hat{T}\right)$ above, the continuity of $\tilde{A}$ follows from the continuity of $\left\langle\Phi\left(q_{1}, q_{2}\right)\left|b^{\dagger} \cdot \Lambda_{e} \cdot b\right| \Phi\left(q_{1}, q_{2}\right)\right\rangle, e \in \mathbb{S}^{2}$, in $q_{1}, q_{2}$. A sufficient condition for $\left\langle\Phi\left(q_{1}, q_{2}\right)\left|b^{+} \cdot \Lambda_{e} \cdot b\right| \Phi\left(q_{1}, q_{2}\right)\right\rangle$ being continuous would be the following growth condition on the coefficients: $\left|c_{l}\right| \leq \frac{C}{T}$ and $\omega_{l} \pm \Omega l \geq l^{1+\epsilon}$ with $\epsilon>0$. While the former condition can be fulfilled with the model parameter describing the molecule-helium interaction, see reference [35], the latter condition can be satisfied by considering a strong magnetic field. Then, $\tilde{A} \underset{q_{1}, q_{2} \rightarrow \mathcal{S}}{\longrightarrow} 0$ vanishes for $q_{1}, q_{2}$ close to the south pole, in contrast to the singular gauge field $\nabla_{j} \phi$ which has a pole at $\mathcal{S}$.

With the convergence $\tilde{A} \underset{q_{1}, q_{2} \rightarrow \mathcal{S}}{\longrightarrow} 0$ at hand, we can verify that the coupling to the background field $\nabla_{j}^{A} \mapsto \nabla_{j}^{A}+i \tilde{A}_{j}$ can be neglected in the limit of large $\Omega$. In order to do this, let us define the dilatation operator

$$
D_{\ell}:\left\{\begin{array}{l}
L^{2}\left(\ell \mathbb{S}^{2}\right) \otimes L^{2}\left(\ell \mathbb{S}^{2}\right) \rightarrow L^{2}\left(\mathbb{S}^{2}\right) \otimes L^{2}\left(\mathbb{S}^{2}\right) \\
\Psi \mapsto D_{\ell}(\Psi)\left(q_{1}, q_{2}\right):=\Psi\left(\ell q_{1}, \ell q_{2}\right) .
\end{array}\right.
$$

Note that the statistics gauge field $A$ transforms exactly like the derivative operator as $D_{\ell}^{-1} A D_{\ell}=\ell A$. Therefore, $D_{\ell}^{-1} \nabla_{j}^{A} D_{\ell}=\ell \nabla_{j}^{A}$ and $D_{\ell}^{-1} H_{\text {Anyon }} D_{\ell}=\ell^{2} H_{\text {Anyon. }}$. Transforming the emerging Hamiltonian yields

$$
D_{\ell}^{-1}\left(\ell^{-2} H_{\text {Emerg }}\right) D_{\ell}=-\sum_{j}\left(\nabla_{j}^{A}+i \ell^{-1} \tilde{A}\left(\ell^{-1} q_{1}, \ell^{-1} q_{2}\right)\right)^{2}+\ell^{-2} \widetilde{V}\left(\ell^{-1} q_{1}, \ell^{-1} q_{2}\right) .
$$

Using the assumption that the confining potential $V$ is quadratic, we see that the natural length scale of the confinement is given by the equation $\ell^{-4} \Omega^{2}$, i.e., $\ell:=\sqrt{\Omega}$. In the next section we will see that a quadratic potential comes naturally, however different choices for $V$ could yield other interesting limits. Since $\ell \rightarrow \infty$ in the limit of large $\Omega$, we conclude $\tilde{A}\left(\ell^{-1} q_{1}, \ell^{-1} q_{2}\right) \rightarrow 0$. With the abbreviation $V_{\Omega}\left(q_{1}, q_{2}\right):=\frac{1}{\sqrt{\Omega}} \widetilde{V}\left(\frac{q_{1}}{\sqrt{\Omega}}, \frac{q_{2}}{\sqrt{\Omega}}\right)$ we then have the asymptotic result

$$
D_{\sqrt{\Omega}}^{-1}\left(\frac{1}{\sqrt{\Omega}} H_{\text {Emerg }}\right) D_{\sqrt{\Omega}} \underset{\Omega \rightarrow \infty}{\longrightarrow} \sqrt{\Omega} H_{\text {Anyon }}+V_{\Omega} .
$$


Hence, in the limit of large $\Omega$, the emerging Hamiltonian corresponds to a system of two anyons living on a sphere of radius $\ell=\sqrt{\Omega}$, with no additional magnetic field but coupled only to an additional scalar potential $V_{\Omega}$.

\section{Realization of a Modified Quantum Dispersion Relation}

We now come back to the standard angulon Hamiltonian (2), and modify it by coupling it to an external magnetic potential $A_{j}^{\Omega}:=\Omega\left(-y_{j}, x_{j}, 0\right)$, i.e., we consider the operator

$$
H_{\text {angulon }, \Omega}:=-\sum_{j}\left(\nabla_{j}-i A_{j}^{\Omega}\right)^{2}+\sum_{l, m} \omega_{l} b_{l, m}^{+} b_{l, m}+b_{Z}^{+}+b_{Z} \text {. }
$$

Let $J_{z}=L_{z, 1}+L_{z, 2}+b^{\dagger} \cdot \Lambda_{z} \cdot b$ be the total angular momentum of the two particles together with the many body environment. By rotating the system in the $x-y$ plane at the cyclotron frequency $\Omega$ we obtain

$$
\begin{aligned}
\tilde{H}_{\text {angulon }, \Omega}: & =e^{-i t \Omega J_{z}}\left(H_{\text {angulon, },}-i \partial_{t}\right) e^{i t \Omega J_{z}}+i \partial_{t} \\
& =-\sum_{j}\left(\left(\nabla_{j}-i A_{j}^{\Omega}\right)^{2}+\Omega L_{z, j}\right)+b^{\dagger} \cdot\left(\omega+\Omega \Lambda_{z}\right) \cdot b+b_{Z}^{\dagger}+b_{Z} \\
& =-\sum_{j} \nabla_{j}^{2}+\Omega^{2} \sum_{j}\left(x_{j}^{2}+y_{j}^{2}\right)+b^{\dagger} \cdot\left(\omega+\Omega \Lambda_{z}\right) \cdot b+b_{Z}^{\dagger}+b_{Z},
\end{aligned}
$$

where we used $\Omega L_{z, j}=-i A_{j}^{\Omega} \cdot \nabla_{j}$. With the definition $V\left(q_{1}, q_{2}\right):=\sum_{j}\left(x_{j}^{2}+y_{j}^{2}\right)$, we see that $\tilde{H}_{\text {angulon, } \Omega}$ almost coincides with

$$
H_{\text {angulon }, \Omega}^{\prime}=-\sum_{j} \nabla_{j}^{2}+\Omega^{2} V\left(q_{1}, q_{2}\right)+b^{\dagger} \cdot\left(\omega+\Omega \Lambda_{\bar{q}}\right) \cdot b+b_{Z}^{\dagger}+b_{Z},
$$

except that the angular momentum operator $\Lambda_{z}$ in $\tilde{H}_{\text {angulon, } \Omega}$ is aligned in the $z$ direction, while for $H_{\text {angulon, } \Omega}^{\prime}$ the operator $\Lambda_{\bar{q}}$ is aligned in the center of mass direction $\bar{q}$.

In the previous section we have seen that the modified angulon Hamiltonian $H_{\text {angulon, } \Omega}^{\prime}$ gives rise to a system of two interacting anyons in the limit of large $\Omega$. In the following, we want to argue why the same conclusion holds for the slightly different operator $\tilde{H}_{\text {angulon, } \Omega \text {, }}$ i.e., we are going to justify that anyons emerge in the low energy regime of $\tilde{H}_{\text {angulon, } \Omega}$ as well. Let us define the Fock space valued function

$$
\Psi:=\exp \left[b_{\left(\omega+\Omega \Lambda_{z}\right)^{-1} Z}-b_{\left(\omega+\Omega \Lambda_{z}\right)^{-1} Z}^{+}\right] \cdot|0\rangle,
$$

which is the vacuum state of the many-body part of $\tilde{H}_{\text {angulon, } \Omega}$, i.e., it is the ground state of

$$
\mathbb{H}_{\Omega}:=b^{\dagger} \cdot\left(\omega+\Omega \Lambda_{z}\right) \cdot b+b_{Z}^{\dagger}+b_{Z} .
$$

In order to observe the emergence of anyons, let us define the alternative section

$$
\Phi:=\exp \left[b_{\left(\omega+\Omega \Lambda_{\bar{q}}\right)^{-1} Z}-b_{\left(\omega+\Omega \Lambda_{\bar{q}}\right)^{-1} Z}^{+}\right] \cdot|0\rangle,
$$

which gives rise to the correct gauge field $A_{z}$. The issue is that $\Phi$ is no longer the vacuum section of $\mathbb{H}_{\Omega}$. However, if we can show that $E_{\Phi}:=\left\langle\Phi\left|\mathbb{H}_{\Omega}\right| \Phi\right\rangle$ approximates the true ground state energy of $\mathbb{H}_{\Omega}$

$$
\tilde{E}_{0}:=-Z^{\dagger}\left(\omega+\Omega \Lambda_{z}\right)^{-1} Z,
$$

and if there is a spectral gap from a nondegenerate ground state, then we can argue that the states (considered as rays of the Hilbert space) are close. 
Let us now verify that the energy deviation $\epsilon:=E_{\Phi}-\tilde{E}_{0}$ is small in the limit of large $\Omega$. First of all, we can express $\epsilon$ as

$$
\epsilon=Z^{\dagger}\left[\left(\omega+\Omega \Lambda_{\bar{q}}\right)^{-1} \Omega\left(\Lambda_{z}-\Lambda_{\bar{q}}\right)\left(\omega+\Omega \Lambda_{\bar{q}}\right)^{-1}-\left(\omega+\Omega \Lambda_{z}\right)^{-1} \Omega\left(\Lambda_{z}-\Lambda_{\bar{q}}\right)\left(\omega+\Omega \Lambda_{\bar{q}}\right)^{-1}\right] Z .
$$

Let us make the reasonable assumption that $\omega+\Omega \Lambda_{\bar{q}}$ is non degenerate, i.e., for simplicity let us assume that $\omega_{l} \pm \Omega l \geq \delta \Omega(l+1)$ for some $0<\delta<1$. Furthermore, we assume that $\left|Z_{l, m}\right| \leq \frac{C}{l+1}$ for some $C$. We define the operator $v$ by $v_{l}:=\sqrt{\delta(l+1)}$ in the diagonalizing basis of $\Lambda_{z}$. Since $\Lambda_{\bar{q}}$ is block-diagonal with respect to $l, \Lambda_{\bar{q}}$ commutes with $v$ and consequently we can rewrite the first part of the error $\epsilon=\epsilon_{1}-\epsilon_{2}$ (the second part can be rewritten in the same way)

$$
\epsilon_{1}=\left(v^{-1} Z\right)^{\dagger}\left[\Omega v^{2}\left(\omega+\Omega \Lambda_{\bar{q}}\right)^{-1} \Omega^{-1} v^{-1}\left(\Lambda_{z}-\Lambda_{\bar{q}}\right) v^{-1}\left(\omega+\Omega \Lambda_{\bar{q}}\right)^{-1} v^{2}\right]\left(v^{-1} Z\right) .
$$

Note that we have the bound on the operator norm $\left\|\Omega v^{2}\left(\omega+\Omega \Lambda_{\bar{q}}\right)^{-1}\right\| \leq 1$, as well

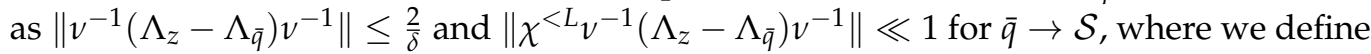
$\chi_{l}^{<L}:=1$ for $l<L$ and $\chi_{l}^{<L}:=0$ otherwise. By our assumption $\left|Z_{l, m}\right| \leq \frac{C}{l}$, we know that $\left\|v^{-1} Z\right\|^{2}=: \widetilde{C}<\infty$, therefore we obtain $\left\|\left(1-\chi_{\ell}^{<L}\right) v^{-1} Z\right\| \underset{L \rightarrow \infty}{\longrightarrow} 0$ and

$$
\begin{aligned}
& \lim _{\bar{q} \rightarrow \mathcal{S}} \Omega \epsilon_{1} \leq \lim _{\bar{q} \rightarrow \mathcal{S}} 2\left(\widetilde{C}\left\|\chi^{<L} v^{-1}\left(\Lambda_{z}-\Lambda_{\bar{q}}\right) v^{-1}\right\|+\frac{2}{\delta}\left\|\left(1-\chi_{\ell}^{<L}\right) v^{-1} Z\right\|^{2}\right) \\
& =\frac{4}{\delta}\left\|\left(1-\chi_{\ell}^{<L}\right) v^{-1} Z\right\|^{2} \underset{L \rightarrow \infty}{\longrightarrow} 0 .
\end{aligned}
$$

Applying a similar argument for $\epsilon_{2}$ yields the estimate for the total error $\epsilon \ll \frac{1}{\Omega}$. Note that the ground state energy itself satisfies $\tilde{E}_{0} \approx \frac{1}{\Omega}$. Consequently, the error term $\epsilon$ is negligibly small even compared to the ground state energy $\tilde{E}_{0}$, i.e.,

$$
\epsilon \ll \tilde{E}_{0} .
$$

We conclude that $E_{\Phi}:=\left\langle\Phi\left|\mathbb{H}_{\Omega}\right| \Phi\right\rangle$ approximates the ground state energy $\tilde{E}_{0}=$ $\left\langle\Psi\left|\mathbb{H}_{\Omega}\right| \Psi\right\rangle$ of $\mathbb{H}_{\Omega}$, and since $\mathbb{H}_{\Omega}$ has a uniform spectral gap this especially means that there exists $\theta_{\bar{q}} \in[-\pi, \pi)$ such that $\left\|\Phi-e^{i \theta_{\bar{q}} \Psi}\right\| \ll 1$. This justifies the usage of the section $\Phi$ instead of $\Psi$ in the Born-Oppenheimer approximation.

Lastly, we stress that the Born-Oppenheimer approximation itself and the emergence of the exact anyonic spectrum, i.e., the spectrum of the Hamiltonian (1), was justified both analytically and numerically for simpler but highly representative models in $[25,35]$.

\section{Conclusions}

In conclusion, we explicitly show that in the Born-Oppenheimer approximation the many-particle bath manifests itself as the statistics gauge field on the two-sphere with respect to the molecular impurities immersed into it.

The analysis of anyons on the sphere becomes much more difficult than the wellstudied planar case, mainly because of non separation of center of mass from relative variables. This problem has also been stressed and tackled in recent studies of anyons on the two-sphere $[30,31,35]$, which have been successful in computing the spectrum of two anyons subject to a homogeneous magnetic field (technically a monopole such that the Dirac quantization condition is satisfied). Our result in this work cannot be directly compared with the explicit spectrum for two anyons computed in $[31,35]$ since it involves additional scalar interactions and a not completely homogeneous magnetic field, and, even more crucially, an effective trapping potential due to the specific rotation setup. Analytical and numerical analyses of the spectra in the present situation are subjects of future work.

We further demonstrate that a possible experimental realization is feasible within the framework of the angulon quasiparticle by applying an external magnetic field to the 
molecular impurities and rotating the impurity-bath system. This lays the foundations for realizing anyons on the two-sphere in terms of molecular impurities in superfluid helium. We finally note that although the dispersion relation of superfluid helium nanodroplets is continuous, the interaction between the molecule and the helium droplet is dominated at a finite excitation momentum, see reference [38]. This allows us to explore the problem with a gapped dispersion relation so that the Born-Oppenheimer approximation can be achieved by considering heavy impurities as discussed in reference [35].

Author Contributions: Writing, M.B. and M.L. and D.L. and E.Y.; funding acquisition, D.L. All authors have read and agreed to the published version of the manuscript.

Funding: This research was funded by Göran Gustafsson Foundation grant number 1804.

Institutional Review Board Statement: Not applicable.

Informed Consent Statement: Not applicable.

Data Availability Statement: Not applicable.

Acknowledgments: D. Lundholm acknowledges financial support from the Göran Gustafsson Foundation (grant no. 1804).

Conflicts of Interest: The authors declare no conflict of interest.

\section{References}

1. Tsui, D.C.; Stormer, H.L.; Gossard, A.C. Two-Dimensional Magnetotransport in the Extreme Quantum Limit. Phys. Rev. Lett. 1982, 48, 1559-1562. [CrossRef]

2. Laughlin, R.B. Anomalous Quantum Hall Effect: An Incompressible Quantum Fluid with Fractionally Charged Excitations. Phys. Rev. Lett. 1983, 50, 1395-1398. [CrossRef]

3. Arovas, D.; Schrieffer, J.R.; Wilczek, F. Fractional Statistics and the Quantum Hall Effect. Phys. Rev. Lett. 1984, 53, 722-723. [CrossRef]

4. Thouless, D.J.; Kohmoto, M.; Nightingale, M.P.; den Nijs, M. Quantized Hall Conductance in a Two-Dimensional Periodic Potential. Phys. Rev. Lett. 1982, 49, 405-408. [CrossRef]

5. Kane, C.L.; Mele, E.J. $Z_{2}$ topological order and the quantum spin Hall effect. Phys. Rev. Lett. 2005, 95, 146802. [CrossRef] [PubMed]

6. Fu, L.; Kane, C.L.; Mele, E.J. Topological Insulators in Three Dimensions. Phys. Rev. Lett. 2007, 98, 106803. [CrossRef]

7. Haldane, F.D.M. Model for a Quantum Hall Effect without Landau Levels: Condensed-Matter Realization of the "Parity Anomaly". Phys. Rev. Lett. 1988, 61, 2015-2018. [CrossRef]

8. Lundholm, D.; Rougerie, N. Emergence of Fractional Statistics for Tracer Particles in a Laughlin Liquid. Phys. Rev. Lett. 2016, 116, 170401. [CrossRef]

9. Kitaev, A. Fault-tolerant quantum computation by anyons. Ann. Phys. 2003, 303, 2-30. [CrossRef]

10. Lloyd, S. Quantum computation with abelian anyons. Quantum Inf. Process. 2002, 1, 13-18. [CrossRef]

11. Freedman, M.; Kitaev, A.; Larsen, M.; Wang, Z. Topological quantum computation. Bull. Am. Math. Soc. 2003, 40, 31-38. [CrossRef]

12. Nayak, C.; Simon, S.H.; Stern, A.; Freedman, M.; Das Sarma, S. Non-Abelian anyons and topological quantum computation. Rev. Mod. Phys. 2008, 80, 1083-1159. [CrossRef]

13. Leinaas, J.M.; Myrheim, J. On the theory of identical particles. Il Nuovo Cimento B 1977, 37, 1-23. [CrossRef]

14. Wilczek, F. Magnetic Flux, Angular Momentum, and Statistics. Phys. Rev. Lett. 1982, 48, 1144-1146. [CrossRef]

15. Wilczek, F. Quantum Mechanics of Fractional-Spin Particles. Phys. Rev. Lett. 1982, 49, 957-959. [CrossRef]

16. Goldin, G.A.; Menikoff, R.; Sharp, D.H. Representations of a local current algebra in nonsimply connected space and the Aharonov-Bohm effect. J. Math. Phys. 1981, 22, 1664-1668. [CrossRef]

17. Wu, Y.S. General Theory for Quantum Statistics in Two Dimensions. Phys. Rev. Lett. 1984, 52, 2103-2106. [CrossRef]

18. Cooper, N.R.; Simon, S.H. Signatures of Fractional Exclusion Statistics in the Spectroscopy of Quantum Hall Droplets. Phys. Rev. Lett. 2015, 114, 106802. [CrossRef]

19. Zhang, Y.; Sreejith, G.J.; Gemelke, N.D.; Jain, J.K. Fractional angular momentum in cold atom systems. Phys. Rev. Lett. 2014, 113, 160404. [CrossRef]

20. Zhang, Y.; Sreejith, G.J.; Jain, J.K. Creating and manipulating non-Abelian anyons in cold atom systems using auxiliary bosons. Phys. Rev. B 2015, 92, 075116. [CrossRef]

21. Morampudi, S.C.; Turner, A.M.; Pollmann, F.; Wilczek, F. Statistics of Fractionalized Excitations through Threshold Spectroscopy. Phys. Rev. Lett. 2017, 118, 227201. [CrossRef] 
22. Umucalılar, R.O.; Macaluso, E.; Comparin, T.; Carusotto, I. Time-of-Flight Measurements as a Possible Method to Observe Anyonic Statistics. Phys. Rev. Lett. 2018, 120, 230403. [CrossRef] [PubMed]

23. Correggi, M.; Duboscq, R.; Lundholm, D.; Rougerie, N. Vortex patterns in the almost-bosonic anyon gas. EPL 2019, 126, 20005. [CrossRef]

24. Yakaboylu, E.; Lemeshko, M. Anyonic statistics of quantum impurities in two dimensions. Phys. Rev. B 2018, $98,045402$. [CrossRef]

25. Yakaboylu, E.; Ghazaryan, A.; Lundholm, D.; Rougerie, N.; Lemeshko, M.; Seiringer, R. Quantum impurity model for anyons. Phys. Rev. B 2020, 102, 144109. [CrossRef]

26. Thouless, D.J.; Wu, Y.S. Remarks on fractional statistics. Phys. Rev. B 1985, 31, 1191-1193. [CrossRef] [PubMed]

27. Einarsson, T. Fractional statistics on a torus. Phys. Rev. Lett. 1990, 64, 1995-1998. [CrossRef] [PubMed]

28. Comtet, A.; McCabe, J.; Ouvry, S. Some remarks on anyons on the two-sphere. Phys. Rev. D 1992, 45, 709-712. [CrossRef]

29. Einarsson, T. Fractional statistics on compact surfaces. Mod. Phys. Lett. B 1991, 5, 675-686. [CrossRef]

30. Ouvry, S.; Polychronakos, A.P. Anyons on the sphere: analytic states and spectrum. Nucl. Phys. B 2019, 949, 114797. [CrossRef]

31. Polychronakos, A.P.; Ouvry, S. Two anyons on the sphere: Nonlinear states and spectrum. Nucl. Phys. B 2020, 951, 114906. [CrossRef]

32. Haldane, F.D.M. Fractional Quantization of the Hall Effect: A Hierarchy of Incompressible Quantum Fluid States. Phys. Rev. Lett. 1983, 51, 605-608. [CrossRef]

33. Harrison, J.M.; Keating, J.P.; Robbins, J.M.; Sawicki, A. n-particle quantum statistics on graphs. Comm. Math. Phys. 2014, 330, 1293-1326. [CrossRef]

34. Maciazek, T.; Sawicki, A. Non-abelian Quantum Statistics on Graphs. Commun. Math. Phys. 2019, 371, 921-973. [CrossRef]

35. Brooks, M.; Lemeshko, M.; Lundholm, D.; Yakaboylu, E. Molecular Impurities as a Realization of Anyons on the Two-Sphere. Phys. Rev. Lett. 2021, 126, 015301. [CrossRef]

36. Schmidt, R.; Lemeshko, M. Rotation of Quantum Impurities in the Presence of a Many-Body Environment. Phys. Rev. Lett. 2015, 114, 203001. [CrossRef] [PubMed]

37. Schmidt, R.; Lemeshko, M. Deformation of a Quantum Many-Particle System by a Rotating Impurity. Phys. Rev. X 2016, 6, 011012. [CrossRef]

38. Lemeshko, M.; Schmidt, R. Molecular Impurities Interacting with a Many-Particle Environment: From Ultracold Gases to Helium Nanodroplets. In Low Energy and Low Temperature Molecular Scattering; Osterwalder, A., Dulieu, O., Eds.; Royal Society of Chemistry: London, UK, 2016.

39. Lemeshko, M. Quasiparticle Approach to Molecules Interacting with Quantum Solvents. Phys. Rev. Lett. 2017, 118, 095301. [CrossRef]

40. Shchadilova, Y. Viewpoint: A New Angle on Quantum Impurities. Physics 2017, 10, 20. [CrossRef]

41. Shepperson, B.; Söndergaard, A.A.; Christiansen, L.; Kaczmarczyk, J.; Zillich, R.E.; Lemeshko, M.; Stapelfeldt, H. Laserinduced alignment of iodine molecules in He-nanodroplets: Long-time coherence, revivals, and breaking-free. arXiv 2017, arXiv:1702.01977.

42. Shepperson, B.; Chatterley, A.S.; Søndergaard, A.A.; Christiansen, L.; Lemeshko, M.; Stapelfeldt, H. Strongly aligned molecules inside helium droplets in the near-adiabatic regime. J. Chem. Phys. 2017, 147, 013946. [CrossRef] [PubMed]

43. Bourdeau, M.; Sorkin, R.D. When can identical particles collide? Phys. Rev. D 1992, 45, 687-696. [CrossRef] [PubMed]

44. Lundholm, D.; Solovej, J.P. Local exclusion and Lieb-Thirring inequalities for intermediate and fractional statistics. Ann. Henri Poincaré 2014, 15, 1061-1107. [CrossRef]

45. Correggi, M.; Oddis, L. Hamiltonians for Two-Anyon Systems. Rend. Mat. Appl. 2018, 39, $277-292$. 